\title{
Grammaticalisation analogue de marqueurs de focalisation en latin tardif et en moyen français ${ }^{1}$
}

\author{
Jesse Mortelmans \\ Université d'Anvers \\ jesse.mortelmans@ua.ac.be
}

\section{Introduction}

Dans cette étude, nous nous proposons de montrer qu'il existe des analogies entre les processus de grammaticalisation d'un type d'expressions référentielles en latin tardif $\left(2^{\mathrm{e}}-6^{\mathrm{e}}\right.$ siècles $)$ et en moyen français $\left(14^{\mathrm{e}}-15^{\mathrm{e}}\right.$ siècles). Il s'agit des marqueurs anaphoriques composés du participe passé d'un verbum dicendi (ou scribendi), renforçant la description définie (marquée par zéro en latin, par le en français), et (souvent) d'un adverbe locatif (supra-, prae-; dessus, devant) signalant que le référent indiqué a déjà été introduit dans le discours. Les premières occurrences des déterminants du type supradictus avaient déjà paru dans des textes en latin classique (attestations dès le $1^{\text {er }}$ siècle avant J.-C.), alors que les premières attestations de le (devant/dessus) dit en ancien français datent du $13^{\mathrm{e}}$ siècle. En latin, il s'agit des formes supra dictus, supra memoratus, praefatus, suprascriptus, praedictus; en français, ce sont avant tout les formes le devant dit, le dessus dit, le dessus nommé ou simplement ledit. En latin tardif comme en moyen français, une évolution similaire se produit : tandis qu'à l'origine l'emploi de ces déterminants était limité à des contextes circonscrits dans des textes administratifs et juridiques, leur fréquence s'élève exponentiellement, l'emploi ne semblant plus être lié à des contextes spécifiques. Des occurrences surgissent également dans des textes littéraires. Cependant, après une période de véritable prolifération d'occurrences, l'emploi est soudainement aboli, sans cause apparente.

Nous avons précédemment mis en relation l'apparition et l'évolution des déterminants du type ledit en moyen français avec les développements au sein du paradigme des démonstratifs (Mortelmans, 2006b). Dans la présente étude, nous mettrons en évidence que pour le latin tardif également l'apparition des déterminants du type supradictus est liée aux évolutions dans le paradigme des démonstratifs. Nous développerons 1'hypothèse que les processus de grammaticalisation au sein des paradigmes des démonstratifs ayant modifié la valeur focalisante de ces déterminants aussi bien en latin tardif qu'en moyen français, les déterminants du type supradictus/ledit reprennent temporairement le rôle focalisant des démonstratifs jusqu'à ce que ces paradigmes soient réorganisés.

Pour le moyen français, c'est surtout la piste de l'influence du style curial qui a souvent été suggérée comme explication pour l'emploi fréquent de ledit (Rasmussen, 1958, Burnley, 1986, Bornstein, 1977). Dans la présente étude, nous invoquerons l'analogie des développements en latin tardif et en moyen français pour amender quelque peu cette hypothèse.

Dans un premier temps, nous (re)tracerons brièvement les évolutions dans l'emploi et le fonctionnement des marqueurs anaphoriques du type supradictus/ledit, puis nous mettrons en évidence, pour les deux périodes concernées, les processus de grammaticalisation des paradigmes des déterminants nominaux, qui semblent jouer un rôle dans ces développements. Finalement, nous déterminerons si les évolutions sont en effet comparables pour les deux langues et, le cas échéant, dans quelle mesure ce constat peut mener à reconsidérer l'hypothèse stylistique. 


\section{Suprascriptus en latin tardif}

Les origines des expressions anaphoriques avec verbum dicendi/scribendi remontent à l'Antiquité grecque et latine. Dans sa grammaire des papyrus grecs de l'époque ptolémaïque ( $\pm 305-30$ avant J.-C.), Mayser (1934) fait mention des participes qui viennent renforcer les articles dans les textes administratifs pour des raisons de clarté et de précision (Mayser, 1934, II, 2 : 11-12). Ces expressions anaphoriques renforcées par des participes

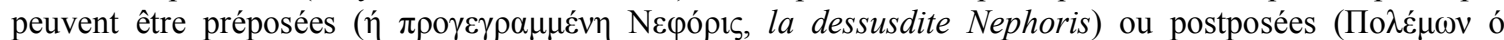
$\pi \rho \gamma \gamma \varepsilon \rho \alpha \mu \mu \varepsilon ́ v o \varsigma$, Polemoon le dessusdit) aux noms qu'ils déterminent (Mayser, 1934, II, 2 : 12).

Plusieurs grammaires latines (Krebs \& Schmalz, 1905, Norberg, 1944, Hofmann \& Szantyr, 1965) signalent qu'à partir du latin classique ( $3^{\mathrm{e}}$ siècle avant J.-C.), mais surtout en latin tardif (à partir du $2^{\mathrm{e}}$ siècle), les expressions supra dictus, supra memoratus, praefatus, suprascriptus, praedictus etc. étaient présentes, voir nombreuses, avant tout dans les écrits administratifs rédigés dans le style de chancellerie et dans la langue des chartes $^{2}$. Selig (1992) donne un aperçu détaillé du développement et de l'évolution des déterminants nominaux en latin tardif à partir de textes juridiques (écrits entre le $2^{\mathrm{e}}$ siècle avant J.-C. et 1200) et hagiographiques (écrits entre 580 et 800). Elle insiste sur la particularité des textes juridiques, dont la priorité absolue est l'assurance de l'univocité du contenu, et qui à cet effet font appel aux moyens jugés redondants dans la communication normale (Selig, 1992: 126). Ainsi, le besoin d'exactitude conduit à un emploi particulier des déterminants dans ces textes : en indiquant clairement si un référent a ou n'a pas encore été introduit dans le discours, les déterminants définis et indéfinis garantissent son identification exacte. Selig remarque que dans les textes juridiques des déterminants apparaissent à des endroits où d'autres textes latins les omettent et confèrent aux éléments du contexte le rôle de résoudre les ambiguïtés possibles (Selig, 1992 : 127). Puisque les textes juridiques font prévaloir le souci de clarté sur les effets stylistiques, ils constituent une source de données importante dans l'étude du développement des déterminants.

Dans la langue juridique et dans les chartes en latin classique, des déterminants anaphoriques s'emploient dans le but d'éviter des malentendus et pour insister sur l'identité exacte des référents : avant tout is, déjà dépourvu de sa force démonstrative et devenu déterminant exclusivement anaphorique, mais également idem (Norberg, 1944 : 70-71). Dans la langue juridique et administrative plus tardive, presque tous les déterminants démonstratifs sont employés de façon anaphorique, sans distinction. Pour insister encore davantage sur le fait que le référent dont il est question a déjà été nommé, il a fallu avoir recours à une description relative (« dont il a déjà été question » ou « qui a déjà été nommé »). Ainsi, les constructions participiales telles que supra dictus et supra nominatus sont entrées dans la langue.

L'aperçu détaillé que donne Selig (1992) de l'ensemble des déterminants en latin tardif contient des chiffres exacts qui illustrent les évolutions dans la fréquence de ce qu'elle appelle les déterminants métalinguistiques. Dans les textes juridiques, la fréquence de supradictus $^{3}$ par rapport aux autres déterminants est de $0,08 \%$ dans les plus anciens textes, augmentant graduellement jusque près de $18 \%$ (dans la tranche de textes de la fin du $5^{\mathrm{e}}$ siècle) et culminant dans la tranche de textes écrits entre 445 et 700 , dans laquelle les déterminants métalinguistiques constituent plus d'un tiers des déterminants. Parallèlement on observe une baisse dans la fréquence du déterminant $i s^{4}$ (de 62,64\% dans la plus ancienne tranche de textes à $12,15 \%$ dans les textes de la fin du $5^{\mathrm{e}}$ siècle) (Selig, $\left.1992: 129\right)$.

Plusieurs documents juridiques du $6^{\mathrm{e}}$ siècle présentent en effet un emploi démesuré des expressions référentielles avec verbum dicendi/scribendi, dans la mesure où les déterminants anaphoriques sont tous remplacés par des expressions anaphoriques comprenant des participes. Norberg (1944: 73) remarque en outre qu'il arrive que les participes s'ajoutent de façon pléonastique aux déterminants anaphoriques, par exemple eidem praefato fundo ou eidem praedictae feminae. Selig (1992) signale le même phénomène : la Doppeldetermination ou la combinaison de déterminants anaphoriques ${ }^{5}$ comprenant souvent un déterminant métalinguistique. 
Tout au long de la période de l'Empire (fin du $1^{\text {er }}$ siècle av. J.-C.-476), les participes sont donc assez fréquents. Par l'influence de l'administration impériale dans les administrations de l'épiscopat et des rois germaniques, ces emplois y ont été repris. L'emploi des participes reste en vigueur dans la chancellerie des Lombards, des Mérovingiens, des Carolingiens et dans les chancelleries médiévales plus tardives ; il constitue une composante essentielle de la forme stéréotypée de l'écriture administrative. Cependant, après le $6^{\mathrm{e}}$ siècle la fréquence des expressions anaphoriques comprenant un verbum dicendi/scribendi commence à décliner, sans toutefois disparaître complètement. Norberg (1944: 72) note que les déterminants idem et ipse prennent parfois le relais ; Selig (1992: 129) remarque surtout une coupure avec la tradition de la fin d'Antiquité à partir des documents juridiques lombards et mérovingiens, qui présentent un pourcentage plus important d'occurrences de ipse par rapport aux déterminants comprenant un verbum dicendi/scribendi.

Norberg (1944) signale finalement que l'emploi des participes combinés à ou remplaçant les expressions anaphoriques est repris dans les nouvelles langues vernaculaires. Il note avant tout la fréquence relativement importante des déterminants antescritto, predetto, prefato, sopradetto, soprascritto dans des textes italiens : leur emploi n'est pas limité à la seule langue administrative, mais se manifeste également dans la littérature plus élevée, par exemple chez Dante (Norberg, 1944 : 73). De même dans d'autres langues vernaculaires, comme le français (voir Bornstein, 1977, Burnley, 1986, De Wolf, 2003, Guillot et al., à paraître et Mortelmans \& Guillot, 2008) et l'anglais (Bornstein, 1977, Burnley, 1986 et Kilpiö, 1997), les expressions anaphoriques avec verbum dicendi/scribendi sont le plus souvent utilisées dans les textes administratifs et juridiques, bien que d'autres genres textuels présentent parfois également de nombreuses occurrences (voir Guillot et al., à paraître).

\section{Ledit en français médiéval}

La question de la fréquence de ledit ${ }^{6}$ dans l'histoire du français a été abordée dans quelques études récentes qui ont trait avant tout au français médiéval. Il a été démontré par De Wolf (2003) et confirmé par Guillot et al. (à paraître) que les premières occurrences du déterminant ledit remontent à la première moitié du $13^{\mathrm{e}}$ siècle. Comme l'a constaté De Wolf (2003 : 337) on ne trouve des occurrences de ce nouveau déterminant à cette époque que dans des chartes et il s'agit au début souvent de variantes plus complexes, comme le devantdit ou le dessusdit. Ces variantes ont existé tout au long de la période de l'ancien et du moyen français, mais, comme l'ont signalé Guillot et al. (à paraître), ces expressions n'ont jamais atteint le même degré de grammaticalisation que ledit. Vers la fin du $13^{\mathrm{e}}$ siècle, des occurrences de ledit, déjà très fréquent dans les chartes à ce moment-là, commencent à apparaître également dans d'autres textes en prose, comme les Coutumes de Beauvaisis de Philippe de Beaumanoir (65 occurrences), texte qui relève néanmoins toujours du registre juridique, et la Vie de Saint Louis de Joinville (19 occurrences) (De Wolf, 2003 : 338).

Ce n'est qu'à partir de l'époque du moyen français, donc à partir du $14^{\mathrm{e}}$ siècle, que ledit fait son entrée véritable dans des textes en prose autres que juridiques ou administratifs (dans lesquels le taux d'occurrences s'élève considérablement); on ne trouve presque pas d'occurrences de ledit dans les textes poétiques ${ }^{7}$. Ledit reste fréquent, toujours en moyen français, dans le domaine d'origine, de même que dans les textes historiques (chroniques et mémoires), allégoriques et didactiques (par exemple le Songe du Viel Pelerin de Philippe de Mézières). L'étude de Guillot et al. (à paraître) a également mis en évidence la présence de ledit dans les textes littéraires de la fin du Moyen Âge, mais cette présence est plus ou moins importante suivant les siècles et suivant les textes : au $14^{\mathrm{e}}$ siècle, les occurrences dans les textes didactiques et littéraires sont plus rares que dans les textes historiques de la même époque; en outre elles ne concernent que quelques œuvres bien déterminées. Au $15^{\mathrm{e}}$ siècle en revanche, ledit est présent de façon beaucoup plus massive à la fois dans les textes historiques et dans les textes didactiques, tandis que son emploi dans la littérature s'étend progressivement, en particulier dans le genre naissant de la nouvelle. La fréquence d'apparition de ledit en moyen français est donc étroitement liée au caractère typologique des œuvres. 
Pour le $16^{\mathrm{e}}$ siècle, l'emploi de ledit est moins bien répertorié. Un aperçu des occurrences dans les textes du $16^{\mathrm{e}}$ siècle compris dans Frantext montre que ledit est toujours relativement fréquent et que son emploi est encore assez répandu dans les différents types de textes. On trouve des occurrences de ledit dans des textes juridiques, dans des traités, des récits de voyage et des chroniques, mais aussi dans des textes littéraires, par exemple chez Rabelais et Montaigne. Quoique certains textes du début du siècle présentent encore un emploi excessif de ledit, comme les Cent nouvelles nouvelles de Philippe de Vigneulles, il s'avère que l'emploi de ce déterminant va nettement déclinant vers la fin du siècle.

Après le $16^{\mathrm{e}}$ siècle, l'emploi de ledit s'effondre assez brusquement; le déterminant a depuis été réservé au registre juridique uniquement, avec par-ci par-là des exceptions, comme le montre l'étude de Whittaker (2003) qui présente une description textuelle et discursive de l'expression anaphorique ledit $N$ en français contemporain.

\section{4. « Marqueurs de focalisation » et grammaticalisation des démonstratifs}

\subsection{La situation en latin tardif}

Comme l'ont signalé Norberg (1944) et Selig (1992), les déterminants avec verbum dicendi/scribendi entrent en concurrence et prennent même le relais des autres expressions anaphoriques, en particulier du déterminant is. Selig (1992) remarque en effet que l'augmentation de la fréquence des déterminants métalinguistiques va de pair avec la baisse de fréquence du déterminant is. Il est étonnant que ce soient les déterminants avec verbum dicendi/scribendi qui relaient is, et non pas idem, ipse ou ille, d'autant plus que, quelques siècles plus tard, c'est bel et bien l'un de ces derniers déterminants qui prend à son tour le relais des déterminants avec verbum dicendi/scribendi dans la plupart des contextes, à savoir ipse. Selon Selig (1992: 129) il ne peut s'agir que d'un phénomène de la langue écrite, la "pédanterie » des expressions anaphoriques avec verbum dicendi/scribendi interdisant leur passage à la communication quotidienne.

Sans expliciter la cause de l'apparition des déterminants métalinguistiques, Selig (1992: 114-120) décrit les développements au sein du paradigme des déterminants démonstratifs qui sont à l'origine des articles (in)définis dans les différentes langues romanes. Il s'avère que le développement des articles (in)définis à partir du latin tardif n'est pas lié exclusivement à la réduction du système casuel latin, comme il a souvent été avancé. En effet, il s'est produit dans le passage du latin aux langues romanes une évolution dans les contextes d'emploi des déterminants, concernant plus particulièrement le degré de focalisation associé aux différentes expressions référentielles impliquées (Selig, 1992 : 117). En latin, l'emploi de déterminants pour signaler la présence en contexte du référent était facultatif. Des noms sans déterminant pouvaient se trouver dans tout contexte pour désigner tout type de référent; ils étaient d'ailleurs largement plus fréquents que les noms avec déterminant. Ceux-ci étaient du coup nettement marqués et liés à des contraintes contextuelles précises. Ils servaient à mettre en évidence d'une façon ou d'une autre la particularité du référent dans le contexte, c'est-à-dire ils fonctionnaient comme des marqueurs de focalisation (Selig, 1992 : 117). Selig distingue en outre deux types de déterminants focalisateurs en latin : d'une part les déterminants emphatiques (hic, iste, ille, idem, ipse), d'autre part les déterminants non-emphatiques (is).

\begin{tabular}{|l|l|l|l|}
\cline { 2 - 4 } \multicolumn{1}{c|}{} & non-focalisation & focalisation $_{1}$ & focalisation $_{2}$ \\
\hline \multirow{4}{*}{ latin } & $\varnothing+\mathrm{N}$ & is $+\mathrm{N}$ & hic $+\mathrm{N}$ \\
& & & iste $+\mathrm{N}$ \\
& & unus $+\mathrm{N}$ & ille $+\mathrm{N}$ \\
& & & ipse + N \\
\hline
\end{tabular}


Cependant, dans le passage du latin aux langues romanes, ce système évolue. Ainsi, en roman, la détermination (in)définie est devenue obligatoire dans les cas de mention spécifique du référent. Le contraste entre les noms déterminés et les noms non-déterminés ne disparaît pas pour autant, mais il ne sert plus à distinguer entre la non-focalisation et la focalisation du référent. Les noms non-déterminés désignent des référents non-spécifiques ou génériques et sont liés à des contraintes contextuelles, de sorte qu'ils sont devenus en roman les formes marquées (Selig, 1992 : 118). Les déterminants non-emphatiques sont "défocalisés» en articles. Selig signale que cette «dé-focalisation» n'entraîne pas pour autant la désémantisation des déterminants ; elle a seulement pour effet de modifier la fonction des déterminants utilisés dans des emplois semblables mais se distinguant au niveau du degré d'emphase (Selig, 1992: 118). À cause de l'extension des contextes d'emploi des déterminants, ceux-ci deviennent plus courants et perdent leur fonctionnement focalisateur. Les déterminants non-emphatiques (is) vont alors occuper les positions qui en latin était réservées à la non-détermination, comme le montre déjà cet extrait des Tabellae Ceratae, rédigées entre 131 et 167 après J.C.

(1) Andueia Batonis emit () domus partem dimidiam (). Eam domus partem dimidiam, qua de agitur, () habere recte liceat. Et si quis eam domum partemve qual quis ex ea evicerit (), tantam pecuniam recte dari fide rogavit Andueia Batonis, (). Proque ea domu partem dimidiam pretium accepisse et abere se dixit. (Tabellae Ceratae VIII, $1-14^{8}$, cité dans Selig, $1992: 128)$

La focalisation ne peut dès lors être réalisée en roman que par le contraste interne entre les déterminants nonemphatiques et les déterminants emphatiques. Dans nombre de langues romanes les déterminants démonstratifs sont alors renforcés par des adverbes locaux, de sorte que la même situation qu'en latin est rétablie. Selig démontre ainsi que le processus de grammaticalisation des déterminants (in)définis latins concerne l'extension de leurs contextes d'emploi, ce qui est d'ailleurs confirmé par Himmelmann (1997) pour d'autres langues également.

\begin{tabular}{|c|c|c|c|}
\hline & non-focalisation & focalisation $_{1}$ & focalisation $_{2}$ \\
\hline roman & article $+N$ & $\begin{array}{l}\text { dém }_{1}+\mathrm{N} \\
\text { dém }_{2}+\mathrm{N} \\
\text { dém }_{3}+\mathrm{N} \\
\text { ident. }+\mathrm{N}\end{array}$ & $\begin{array}{l}\text { dém } 1+\mathrm{N}+\text { adv. } \\
\text { dém } 2+\mathrm{N}+\text { adv. } \\
\text { dém } 3+\mathrm{N}+\text { adv. }\end{array}$ \\
\hline
\end{tabular}

Tableau 2 : distribution des marqueurs de focalisation en roman (d'après Selig, $1992: 119$ )

C'est précisément lorsque l'on n'arrive plus à exprimer l'opposition entre les deux types de focalisation que surgissent les nombreuses occurrences des déterminants avec verbum dicendi/scribendi. Étant donné le caractère explicitement anaphorique de ces déterminants, on pourrait conclure que ceux-ci viennent combler temporairement le vide référentiel causé par la transition des marqueurs de focalisation.

Selig (1992: 131-132) stipule en outre que la raison pour laquelle dans les textes juridiques is est plutôt remplacé par les déterminants métalinguistiques que par idem ne réside pas dans la nature purement anaphorique de ce dernier déterminant. En effet, les déterminants métalinguistiques thématisent le fait que le référent désigné a déjà été introduit dans le discours ; ils sont dès lors aussi explicitement anaphoriques que idem. Ceci vaut d'ailleurs aussi pour ipse, puisque c'est ce déterminant qui viendra à son tour prendre le relais des déterminants métalinguistiques dans la plupart des contextes. Ce qui unit ces déterminants explicitement anaphoriques c'est qu'ils se distinguent des «groupes nominaux définis restrictifs » qui désignent les premières occurrences des référents dans le discours. Ceux-ci sont déterminés par ille ${ }^{9}$. C'est dans cette 
répartition des contextes d'emploi que se situe selon Selig (1992 : 132) la clé de l'évolution des déterminants nominaux.

\subsection{La situation en français médiéval}

Tout comme c'était le cas en latin tardif, l'apparition des déterminants du type ledit en moyen français coïncide avec des évolutions fondamentales au sein du paradigme des démonstratifs. Comme l'a démontré Marchello-Nizia (2001, 2003a, 2004, 2005, 2006a, b, c et passim), il y a eu dans l'histoire du français une période dans laquelle la portée sémantique des démonstratifs était brouillée à cause d'un double bouleversement, formel et sémantique, de leur système. En effet, du point de vue sémantique, on peut distinguer avant le moyen français trois états successifs. Selon la répartition classique, l'opposition sémantique entre les trois paradigmes en latin hic, iste et ille était avant tout de nature "personnelle », et secondairement évaluative et spatiale (hic visant l'objet présent ou celui qui concerne le sujet parlant, iste visant l'objet intéressant l'interlocuteur, ou exprimant une connotation dépréciative et ille visant l'objet éloigné et, secondairement une personne ou un objet connu ou notoire) (Väänänen, 1967).

Le très ancien français n'a conservé que deux de ces paradigmes, sous leurs formes renforcées (ecce $+i$ iste et ecce + ille) comme pronom et déterminant, qui s'opposaient nettement du point de vue sémantique ${ }^{10}$. A partir de certaines constructions fréquentes où les démonstratifs référaient à un discours qui venait d'être tenu (cist référant à ces paroles si elles venaient du locuteur, cil étant employé dans les autres cas), une nouvelle valeur spatiale, autoréférentielle, s'est développée, qui a comme point de référence l'occurrence elle-même (voir Guillot, 2003b et 2005, Kleiber, 1985 et 1987 et Marchello-Nizia, $1995:$ 130, 2005 et 2006a).

Parallèlement, au plan morphologique, un nouveau paradigme de déterminants ce / ces, neutralisé du point de vue des oppositions sémantiques, s'est développé dès 1200. Les nouvelles formes de ce troisième paradigme s'étaient ajoutées à la gamme de formes des paradigmes cist et cil, parmi lesquelles il faut compter également les «formes longues », à savoir les formes suffixées en $-u i$ ou $-i$, ou préfixées en $i$-. Il en a résulté une véritable panoplie de formes pour le moyen français, période dans laquelle le système des démonstratifs était en pleine transition et les nouvelles oppositions sémantiques n'étaient pas encore articulées. Le rôle exact des démonstratifs en moyen français n'était donc pas très clairement déterminé, leur portée étant brouillée par les évolutions qui s'étaient produites tant au plan formel qu'au plan sémantique ${ }^{11}$.

Dans la littérature sur les déterminants en moyen français, les rares descriptions de ledit que nous possédons insistent généralement sur sa valeur désambiguïsante. En effet, le rôle désambiguïsant de ledit dans son domaine d'origine, les chartes et les écrits juridiques, est évident : dans ces textes, le déterminant sert à bien distinguer les différents référents, afin d'éviter les équivoques qui pourraient avoir des conséquences juridiques importantes.

(2) Si prions a noble dame et sage madame Mahaut, castellaine de Bruxelle, et a noble damoisiaul Gerart de Marbais, fil a le dite castellaine, desqueis nos tenons nostre fief de Seneffe, qu'il toutes les choses ki sont contenues en ces presentes chartres loent [...] (Charte 277 de l'abbaye de Ninove, 1290, Fonds de Smet, transcription par Peersman, 2003)

Dans les textes littéraires en moyen français, ledit semble initialement maintenir son rôle désambiguïsant: Jokinen (1992 : 56-57) considère que les "moyens discursifs" tels que ledit servent à soutenir les structures grammaticales "hésitantes". De même, Perret (2000: 20) postule que l'absence d'anaphoriques sélectifs comme celui-ci ou ce dernier, qui n'étaient pas encore en usage, est à l'origine des nombreuses reprises immédiates en moyen français par un groupe nominal anaphorique identique à celui de la désignation précédente du référent, parfois soulignées par ledit, qui ont souvent pour objet de lever l'ambiguïté possible entre deux référents. 
Cependant, il a été mis en évidence que le fonctionnement textuel de ledit ne se limite pas à la désambiguïsation : ledit sert en outre comme marqueur de focalisation, et rejoint en cela certains aspects du fonctionnement textuel des démonstratifs ${ }^{12}$ (Guillot, 2003a, Mortelmans, 2006b, Mortelmans et Guillot, 2008).

Et disoient auqunes gens que les secrés de ces parlemens estoient par lui sceu en France, car il avoit un sien chevalier le plus secré de tous les aultres et que le plus il amoit, lequel on nonmoit messire Lois de Cranehen, envoiiet a Paris, et la se tenoit tous quois dalés le roi et les signeurs. Et estoit li dis messires Loys cargiés de par le duc [...]. (Froissart, p. 292)

(4) Et vint li rois d'Engleterre a Louvaing et se loga ou chastiel, car li dus son cousin li presta. Et manda li dis rois d'Engleterre la roine Phelippe sa fenme en Engleterre, [...]. (Froissart, p. 295-296)

(5) Et comme ledict conte fut en train, le roy vint à luy et le conduysit jusques à Villiers le Bel, qui est ung villaige à quatre lieues près Paris, monstrant par effect avoir ung grant desir de l'amitié dudict conte, et tous deux y logèrent ce soir (Commynes I, p. 87)

(6) En la duché de Bourgoigne eut nagueres ung gentil chevalier dont l' ystoire presente passe le nom, qui maryé estoit a une belle et gente dame. Assez près du chasteau ou le dit chevalier faisoit sa residence demouroit ung musnier [...] (Cent nouvelles nouvelles, p. 37)

D'un côté ledit permet de résoudre l'ambiguïté locale, mais d'un autre côté ce déterminant sert souvent à focaliser l'attention du lecteur sur les référents (déjà ou devenus) cognitivement saillants (cf. Mortelmans et Guillot, $2008: 321)$.

Ledit ne prend évidemment pas en charge tous les aspects du fonctionnement textuel des démonstratifs, mais il s'avère qu'il vient au renfort pour ce qui concerne la désambiguïsation et la focalisation, deux emplois typiques des démonstratifs temporairement éliminés par les développements au sein du paradigme en transition. Nous pouvons en conclure qu'en moyen français comme en latin, les déterminants à verbum dicendi/scribendi ont été introduits pour combler un certain vide référentiel causé par les transitions dans le paradigme des démonstratifs.

\section{La grammaticalisation des déterminants anaphoriques à verbum dicendil scribendi}

Quelques éléments semblent suggérer que les expressions anaphoriques à verbum dicendi/scribendi ont ellesmêmes subi une grammaticalisation après leur intégration dans le paradigme des déterminants nominaux. Ainsi, la fréquence élevée de supradictus vers la fin de la période du latin tardif et de ledit vers la fin de la période du moyen français met en évidence que les contextes dans lesquels ils sont utilisés sont plus nombreux que lorsqu'ils étaient initialement introduits dans la langue. Comme il a été signalé ci-dessus, Norberg (1944: 73) a noté que certains documents du $6^{\text {e }}$ siècle présentent en effet un emploi illimité des expressions référentielles avec verbum dicendi/scribendi, dans la mesure où les déterminants anaphoriques sont tous remplacés par des expressions anaphoriques comprenant des participes. De même, plusieurs textes de la fin de la période du moyen français (début du $16^{\mathrm{e}}$ siècle), par exemple les Mémoires de Philippe de Commynes ou les Cent nouvelles nouvelles anonymes, présentent de très nombreuses occurrences dans des contextes très divers. Dans son étude sur la prose narrative du $15^{\mathrm{e}}$ siècle, Rasmussen $(1958: 33-34)$ remarque que l'emploi de ledit «est si fréquent dans certains textes que le terme devient presque l'équivalent de l'article défini. » A l'origine, les déterminants à verbum dicendi/scribendi étaient introduits dans la langue dans un but bien précis de désambiguïsation. Les déterminants avaient initialement le rôle de marquer la reprise anaphorique explicite en référence intradiscursive, et dans ce contexte ils rejoignaient à l'origine le fonctionnement textuel des démonstratifs. Or, leur emploi s'étend assez vite à d'autres contextes, selon 
l'évolution qui marque tout processus de grammaticalisation de démonstratifs (Himmelmann, 1997, Diessel, 1999).

Par ailleurs, le critère de l'agglutination ${ }^{13}$ révélateur des processus de grammaticalisation (Lehmann, 1985/2002 : 135) constitue un second indice. En ce qui concerne les graphies des textes anciens et médiévaux, il faut bien entendu prendre en compte que le texte édité est le résultat des choix de l'éditeur. Il est dès lors plutôt risqué d'invoquer la graphie de textes édités pour étayer son raisonnement, mais certaines tendances nettes et facilement vérifiables dans les manuscrits (ou sur d'autres sources matérielles) permettent pourtant d'avancer des hypothèses concernant la graphie des déterminants en question. Ainsi, en latin tardif, la fréquence des abréviations ss met en évidence à quel point l'emploi des déterminants avec verbum dicendi/scribendi était généralisé, comme le montre par exemple cette charte de dotation de l'année $252^{14}: \ldots$ monumentum... inque vacuam possessionem monumenti ss (=supra scripti)... ad id monumentum... monumentum ss (cité dans Norberg, 1944 : 71). De même, les abréviations sstus, ssta, sstum etc. étaient fréquentes à l'époque plus tardive. En ce qui concerne le français, le passage en revue de l'emploi de ledit à différentes époques dans l'histoire de la langue met en évidence la diversité des graphies possibles. Dans les plus anciens textes, ledit se compose clairement de deux éléments séparés, d'une part l'article défini, et d'autre part le participe passé du verbe dire. En revanche, dans les manuscrits des textes du début du $16^{\mathrm{e}}$ siècle, pratiquement toutes les formes de ledit sont agglutinées à tel point qu'on trouve même des abréviations $\left(l e^{d}\right.$ ou $\left.l e s^{d}\right)$. Ainsi, les composantes des déterminants appartenant à des catégories grammaticales différentes (adverbe locatif et participe en latin tardif; article défini et participe en français médiéval) se sont jointes pour former un déterminant agglutiné à part entière qui constitue un marqueur référentiel distinct.

\section{Question de stylistique ?}

Les études littéraires qui abordent le curieux phénomène de l'emploi de plus en plus fréquent de ledit dans les textes en moyen français, surtout au $15^{\mathrm{e}}$ siècle, partent tous du principe qu'il a ses origines dans le style curial, le style de prose issu de la chancellerie du Moyen Âge, qui fut élaboré par les clercs de la curia Romana, l'administration centrale de l'église catholique (Rasmussen, 1958 : 32). Bien que ce style fût développé en latin, ses traits ont été transposés aux langues vernaculaires. En fait, le style curial n'est pas en soi un style littéraire; l'appellation désigne plutôt une série de caractéristiques formelles (expression formulaires, marqueurs référentiels avec verbum dicendi/scribendi, mots latins, explications élaborées, binômes ou trinômes synonymiques, etc. (Bornstein, 1977: 370)) employées dans les documents juridiques et diplomatiques dans un but fonctionnel de précision de la référence et du ton solennel et cérémoniel. Il va sans dire que la prose en moyen français est profondément marquée par ces traits, et que certains auteurs, surtout vers la fin de la période du moyen français, s'en servent à tort et à travers dans le but d'accorder à leurs récits un maximum d'authenticité (voir Mortelmans 2006a). C'est ainsi que les éléments issus de la langue administrative et juridique perdent toute leur valeur significative de balisage de discours et sont réduits à des marques purement formelles.

Quoique vers la fin de la période du moyen français l'emploi des déterminants avec verbum dicendi/scribendi soit donc plutôt inspiré par un souci de formalisme, l'analogie avec les développements en latin tardif met en évidence qu'à l'origine l'introduction de ces déterminants dans la langue doit être liée aux évolutions au sein du paradigme des démonstratifs. En outre, dans la plupart des textes en moyen français, l'emploi de ledit n'est pas assez aléatoire pour le considérer comme une marque purement formelle; il est au contraire possible de distinguer certaines régularités - que nous avons décrites ailleurs (Mortelmans \& Guillot 2008) - qui montrent que ledit a effectivement une fonction circonscrite au sein du discours.

Notons encore que le français médiéval n'est pas la seule langue vernaculaire pour laquelle les fréquences d'une expression comme ledit ont été répertoriées. En établissant l'aperçu des expressions du type aforesaid en ancien et moyen anglais, Kilpiö (1997) a démontré que l'anglais médiéval présente des tendances 
semblables au français médiéval pour ce qui est de l'évolution des fréquences et de la répartition dans les genres textuels. Ces déterminants ont sans doute été introduits dans la langue sous l'influence du français (voir Mustanoja, 1960 : 177 et Kilpiö, 1997 : 80, et plus indirectement Bornstein, 1977 et Burnley, 1986). Les expressions du type aforesaid deviennent fréquentes dans la langue anglaise vers 1350-1420, la période entre 1420 et 1500 constituant leur apogée, lorsque les occurrences sont non seulement nombreuses mais en outre réparties sur une variété de types de textes. Quoique toujours assez fréquent aux débuts de la période moderne (Early Modern English), l'emploi des expressions participiales décline au $16^{\mathrm{e}}$ siècle pour être associé de plus en plus exclusivement au registre juridique. Cette évolution pourrait constituer un argument en faveur de l'hypothèse stylistique. L'influence du moyen français avec toutes ses particularités est en effet tellement importante pour le moyen anglais, que dans ce cas cette hypothèse serait bien justifiée. En revanche, l'histoire du français n'est pas l'histoire de l'anglais ni de quelque autre langue. Il s'avère que l'analogie dans le rôle des déterminants avec verbum dicendi/scribendi au sein de l'ensemble des déterminants nominaux ne vaut pour l'instant que pour les développements en latin tardif et en moyen français.

\section{Bilan et perspectives}

En établissant l'analogie dans l'apparition et l'évolution des déterminants avec verbum dicendi/scribendi en latin tardif et en moyen français, nous avons démontré que des développements au sein du paradigme des démonstratifs sont à l'origine de l'emploi de supradictus/ledit. Ces déterminants viennent d'abord combler le vide référentiel qui est la conséquence directe des transitions des démonstratifs, pour ensuite faire partie intégrante des déterminants nominaux pendant une certaine période, jusqu'à ce que le paradigme des démonstratifs se réorganise, rendant superflu l'emploi des expressions anaphoriques avec verbum dicendi/scribendi. Dès lors, on peut conclure que des causes autres que stylistiques peuvent être à l'origine de l'emploi fréquent de ledit en moyen français.

Afin de vérifier ces conclusions, il faudrait mener des recherches sur les développements des déterminants nominaux dans les autres langues vernaculaires également qui mettent en œuvre des déterminants avec verbum dicendi/scribendi.

\section{Références bibliographiques}

\section{Textes}

Base de français médiéval : http://bfm.ens-lsh.fr.

Base textuelle Frantext : http://atilf.atilf.fr/frantext.htm.

Cent nouvelles nouvelles, édité par SWEETSER, F. P. (1966). Genève : Droz.

Jean FROISSART, Chroniques, édité par DILlER, G. T. (1972) (édition du manuscrit de Rome Reg. lat. 869). Genève : Droz.

PEERSMAN, C. (2003) Les chartes en ancien français antérieures à 1300 de l'abbaye de Ninove. Édition et analyse, Mémoire de licence, KULeuven.

Philippe de COMMYNES, Mémoires, édité par CALMETTE, J. (1964-1965). Paris: Belles Lettres (Classiques de l'Histoire de France au Moyen Âge). 


\section{Études}

Bornstein, D. (1977). French influence on Fifteenth-Century English Prose as exemplified by the translation of Christine de Pizan's Livre du Corps de Police. Mediaeval Studies, 39, 369-386.

Burnley, J. D. (1986). Curial Prose in England. Speculum, 61/3, 593-614.

De Wolf, A. (2003). Un nouveau déterminant : le déterminant anaphorique -dit en français médiéval. Verbum, 25/3, 335351.

Diessel, H. (1999). Demonstratives: form, function, and grammaticalisation. Amsterdam : John Benjamins.

Dees, A. (1971). Etude sur l'évolution des démonstratifs en ancien et moyen français. Groningue : Wolters-Noordhoff.

Guillot, C. (2003a). Grammaticalisation et système de la référence : Cellui, icellui, cest, cestui et ledict dans un texte du début du $\mathrm{XV}^{\mathrm{ème}}$ siècle. Verbum, 25/3, 369-379.

Guillot, C. (2003b). Le rôle du démonstratif dans la cohésion textuelle au XVìme siècle. Eléments de grammaire textuelle. Thèse de doctorat, Lyon : ENS-LSH.

Guillot, C. (2005). "Ceste parole" et "ceste aventure" dans la Queste del saint graal. Marques de structuration discursive et transitions narratives. L'information grammaticale, 105, 23-27.

Guillot, C. et al. (à paraître). Typologie des textes et des phénomènes linguistiques pour l'analyse du changement linguistique avec la Base de Français Médiéval. In Actes de Corpus et questionnements du littéraire (Université de Paris $X, \quad$ novembre 2005). Déjà disponible sur http://weblex.enslsh.fr/biblio/slh/GuillotHeidenLavrentievTypologieAnalyseLEDIT.doc

Himmelmann, N. P. (1997). Deiktikon, Artikel, Nominalphrase. Zur Emergenz syntactischer Struktur. Tübingen: Max Niemeyer.

Hofmann, J. B. \& Szantyr, A. (1965). Lateinischen Syntax und Stilistik. München: C. H. Beck’sche Verlagsbuchhandlung.

Jokinen, U. (1992). Réflexions sur l'anaphore en moyen français. Travaux de linguistique, 25, 47-58.

Kilpiö, M. (1997). Participial Adjectives with Anaphoric Reference of the Type the said, the (a)forementioned from Old to Early Modern English: The Evidence from the Helsinki Corpus. In Nevalainen, T. \& Kahlas-Tarkka, L. (éds), To Explain the Present. Studies in the Changing English Language in Honour of Matti Rissanen, Helsinki: Société néophilologique, 77-100

Kleiber, G. (1985). Sur la spécialisation grammaticale des démonstratifs du français ancien. Verbum, numéro spécial, 99113

Kleiber, G. (1987). L'opposition cist/cil en ancien français ou comment analyser les démonstratifs ?. Revue de linguistique romane, 51, 5-35.

Krebs, J. Ph. \& Schmalz, J. H. (1905). Antibarbarus der Lateinischen Sprache. Basel: Benno Schwabe.

Lehmann, C. $\left(1985 / 2002^{2}\right)$. Thoughts on grammaticalization. Second, revised edition. Universität Erfurt: Seminar für Sprachwissenschaft.

Marchello-Nizia, C. (1995). L'évolution du français. Ordre des mots, démonstratifs, accent tonique. Paris : Armand Colin.

Marchello-Nizia, C. (2001). Grammaticalisation et évolution des systèmes grammaticaux. Langue française, 130, 33-41.

Marchello-Nizia, C. (2003a). "Se voz de ceste ne voz poez oster, je voz ferai celle teste coper" (Ami et Amile 753) : La sphère du locuteur et la deixis en ancien français. In De Wilde, P., et al. (éds), Mémoire en temps advenir. Hommage à Theo Venckeleer, Louvain : Peeters, 413-427.

Marchello-Nizia, C., (2003b). Changes in the structure of grammatical systems: The evolution of French. Forum for Modern Language Studies, 39/4, 371-385. 
Marchello-Nizia, C. (2004). La sémantique des démonstratifs en ancien français : une neutralisation en progrès ?. Langue française, 141, 69-84.

Marchello-Nizia, C. (2005). Deixis and subjectivity : the semantics of demonstratives in Old French (9th-12th century). Journal of Pragmatics, 37, 43-68.

Marchello-Nizia, C. (2006a). Du subjectif au spatial : l'évolution des formes et du sens des démonstratifs en français. Langue française, 152/4, $114-126$.

Marchello-Nizia, C. (2006b). Grammaticalisation et changement linguistique. Bruxelles : De Boeck.

Marchello-Nizia, C. (2006c). From personal deixis to spatial deixis: the semantic evolution of demonstratives from Latin to French. In Hickmann, M. \& Robert, S. (éds), Space in Languages: Linguistic Systems and Cognitive Categories, Amsterdam, Philadelphie : John Benjamins, 103-120.

Mayser, E. (1934). Grammatik der Griechischen Papyri aus der Ptolemäerzeit. Berlin/Leipzig: Walter De Gruyter \& Co.

Mortelmans, J. (2006a). Escrire et mettre par memoire. La fausse objectivité dans les chroniques en moyen français. In: Van Hemelryck, T. \& Van Hoorebeeck, C. (éds), L'écrit et le manuscrit à la fin du Moyen Âge. Actes du 2e colloque international du GRMF (Louvain-la-Neuve, 12-14 mai 2005), Turnhout : Brepols, 239-250.

Mortelmans, J. (2006b). Ledit vs le démonstratif en moyen français: quels contextes d'emploi ?. Langue française, 152, 70-81.

Mortelmans, J. \& Guillot, C. (2008). Clarté ou vérité ? LEDIT dans la prose de la fin du Moyen Âge. In Bertrand, O. et al. (éds), Discours, diachronie, stylistique du français. Études en hommage à Bernard Combettes, Berne : Peter Lang, 307-323.

Mustanoja, T. F. (1960). A Middle English Syntax. Part I: Parts of Speech. Helsinki: Société Néophilologique.

Norberg, D. (1944). Beiträge zur Spätlateinischen Syntax. Uppsala: Almqvist \& Wiksells Boktryckeri AB.

Perret, M. (2000). Quelques remarques sur l'anaphore nominale aux XIVe et XVe siècles. L'information grammaticale, $87,17-23$.

Price, G. (1968). Quel est le rôle de l'opposition cist/cil en ancien français ?. Romania 89/2, 240-253.

Rasmussen, J. (1958). La prose narrative française du $X V^{e}$ siècle. Etude esthétique et stylistique. Copenhague, Munksgaard.

Selig, M. (1992). Die Entwicklung der Nominaldeterminanten im Spätlatein. Tübingen : Gunter Narr.

Väänänen, V. (1967). Introduction au latin vulgaire. Paris : Klincksieck.

Whittaker, S. (2003). Pour une description textuelle et discursive de l'expression anaphorique ledit N. French Language Studies, 13, 159-176.

\footnotetext{
${ }^{1}$ Nous souhaitons exprimer notre gratitude envers Walter De Mulder pour ses relectures critiques et ses précieux conseils que nous avons essayé d'intégrer. Les maladresses qui subsistent sont de notre fait.

Une version abrégée de cet article paraîtra dans L'information grammaticale, dans le numéro thématique sur les Propriétés énonciatives et pragmatiques du texte médiéval coordonné par Amalia Rodriguez Somolinos et Evelyne Oppermann-Marsaux (prévu pour juin 2008).

${ }^{2}$ Dans la littérature, la première occurrence de supra scriptus apparaît dans Vitruve (1er siècle avant J.-C.) in dextra parte fanum est Veneris et fons supra scriptus. Pour praedictus et supra dictus, des occurrences sont trouvées dans Columelle, Pline l'Ancien, Quintilien (1er siècle après J.-C.) et Tacite (1er-2e siècle) (voir Norberg, 1944 : 71).

${ }^{3}$ Par supradictus nous désignons dorénavant l'ensemble des expressions référentielles avec verbum dicendi/scribendi en latin tardif (supradictus, praefatus, supra nominatus, supra memoratus, suprascriptus, praedictus etc.).
} 
${ }^{4}$ La fréquence du pronom is par contre reste assez élevée jusque dans les textes du haut Moyen Âge.

${ }^{5}$ Selig (1992: 137) remarque que les seuls déterminants qui ne sont jamais combinés entre eux dans une telle Doppeldetermination sont ille et ipse.

${ }^{6}$ Par la suite, nous désignerons par ledit l'ensemble des différentes graphies de ce déterminant en moyen français.

${ }^{7}$ Il est toutefois intéressant de noter sous ce rapport que dans les mises en prose de textes en vers on trouve des occurrences de déterminants en - dit à des endroits où dans le texte en vers se trouve un déterminant zéro ou un article défini (De Wolf, $2003: 341$ ).

${ }^{8}$ Corpus Inscriptionum Latinarum (Berlin) III, 2, pp. 921-960.

${ }^{9}$ Le déterminant ille peut bien entendu être anaphorique également, mais à la différences des autres anaphoriques, ille $+N$ a la particularité de pouvoir désigner les premières occurrences des références.

${ }^{10}$ La nature exacte de cette opposition sémantique a fait l'objet de mainte étude (voir Kleiber 1987, qui démontre que les modèles développés par (entre autres) Price, 1968 et Dees, 1971 ne s'avèrent pas suffisants pour rendre compte de la totalité des occurrences de cist et cil ; voir aussi les travaux de Marchello-Nizia, 2003a, 2004, 2005 et 2006a, b et c qui remettent en partie en question l'analyse de Kleiber).

${ }^{11}$ Toutefois, Guillot (2003b) a démontré que malgré la grande variété de formes, le démonstratif a une fonction textuelle essentielle dans les textes en moyen français, et qu'il permet de structurer et de segmenter le discours (Guillot, 2003b : 13).

${ }^{12}$ Walter De Mulder (cp) nous signale que cet effet de focalisation n'a pourtant pas nécessairement la même origine que chez les démonstratifs ; il découle peut-être tout simplement de la précision apportée par «-dit ».

${ }^{13}$ Par la notion d'agglutination, Lehmann désigne la phase dans le processus de coalescence de deux éléments juxtaposés dans laquelle un élément devient un affixe de l'autre (Lehmann, 1985/2002 : 132).

${ }^{14}$ Corpus Inscriptionum Latinarum (Berlin) VI 10247, cité dans Norberg (1944: 71). 